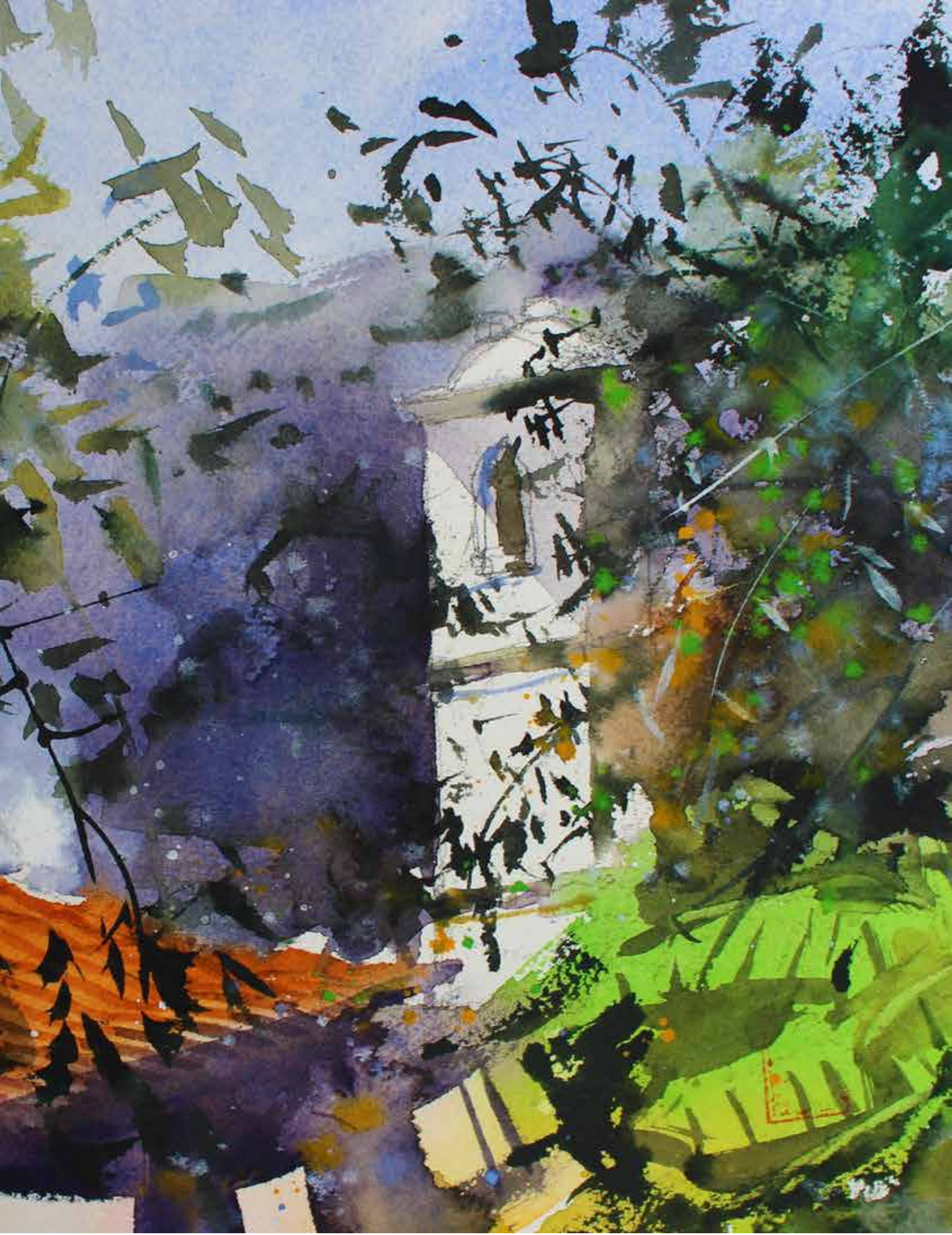




\section{Fecundidad}

iMujeres! La belleza es una forma y el óvulo una idea...

iTriunfe el óvulo!

Dentro de la mentira de la vida

existe una verdad

y bay que seguirla.

La verdad es que nada en la Natura debe perderse.

La tierra que es moral porque procrea abre la entraña a la simiente y brota dándonos trigo.

El vientre que se da sin reticencias pone un soplo de Dios en su pecado.

Son para él las rosas que abre el sol. El vibrará como una cuerda loca que el misterio estremece.

El vientre que se niegue será atado al carro de la sed eternamente.

iMujeres! Sobre el grito de lo bello grite el impulso fuerte de la raza. iCada vientre es un cofre!

¿Qué se guarda en las células que tiene? ¿Cuántos óvulos viejos ban rodado guardándose el misterio que encerraban?...

¿Estaba en ellos quien bacía falta? iMujeres! La belleza es una forma y el óvulo una idea.

Alfonsina Storni

\section{Ulises Rivera}

Técnica

Acuarela 


\title{
7. Determinantes próximos de la fecundidad adolescente en Honduras período 2011-2012
}

\author{
Jinna Yennsy Rosales ${ }^{1}$ \\ DOI: https://doi.org/10.5377/pdac.v15i0.8118 \\ Recibido: 02/05/2019 - Aceptado: 23/05/2019
}

\begin{abstract}
Resumen: Este artículo tiene como objetivo analizar las determinantes indirectas e intermedias relacionadas con la fecundidad adolescente y su vinculación con el nivel de escolaridad. Metodológicamente se replica el modelo de fecundidad planteado por John Bongaarts; utilizando los datos de la Encuesta de Demografía y Salud (ENDESA) 2011-2012. Tras examinar las variables intermedias que plantea el modelo, se identificó como la variable más influyente la nupcialidad o unión temprana; en un período de tres años la tasa global de fecundidad adolescente fue de 1.46, valor que presenta el promedio de hijos por cada mil mujeres adolescentes, además se encontró que en los casos de adolescentes embarazadas o que ya eran madres para el año de la encuesta, la mayoría se encontraba residiendo en el área rural. Estas adolescentes presentan características sociodemográficas diferentes a las del área urbana como: mayor número de hijos, menor nivel de escolaridad, y se ubican en los quintiles más bajos de riqueza.
\end{abstract}

Palabras clave: fecundidad adolescente, pobreza, variables intermedias.

\section{Future determinants of the adolescent fertility in Honduras period 2011-2012}

Abstract: The purpose of this article is to analyze the indirect and intermediate determinations related to adolescent fertility and its relationship with schooling level. Methodologically, replicating the fertility model proposed by John Bongaarts; and using data from the Demographic and Health Survey (ENDESA) 2011-2012. After examining the intermediate variables posed by the model, nuptiality or early union was identified as the most influential variable. In a period of three years, the global rate of adolescent fertility is 1.46 , which is the average of children per thousand in adolescent women. In addition, it was found that in the cases of pregnant adolescents or those who were already mothers during the year of the survey, the majority were residing in the rural area. These adolescents present sociodemographic characteristics different from those of the urban area such as greater number of children, lower level of schooling, and in the lowest wealth quintile.

Keywords: adolescent fertility, poverty, intermediate variables.

\section{Introducción}

La fecundidad adolescente es un problema de carácter social, económico y demográfico que se refleja en la interrupción del desarrollo y la evolución normal de los rasgos biopsicosociales de la mujer adolescente. Es un fenómeno que se manifiesta a nivel mundial, dejando como consecuencia una seria preocupación por la incidencia de casos desfavorables o por las complicaciones que se presentan para la madre adolescente y el feto.

En fecundidad, diferentes factores han sido estudiados para explicar el nivel y las diferencias entre poblaciones. Se habla de determinantes indirectos (tales como la mortalidad infantil, la urbanización y el nivel educativo) y de determinantes próximos o intermedios (por ejemplo, la nupcialidad y el uso de anticonceptivos), a través de los cuales cualquier factor social influye en el nivel de la fecundidad (Guiomar, 2003, pág. 7).

Una de las consecuencias de la fecundidad adolescente es el abandono de la educación formal, sobre todo en las adolescentes de escasos recursos, y por tanto la pérdida inmediata de las posibilidades de lograr un mejor estatus social a lo largo de sus vidas.

En Honduras predominan los prejuicios y tabúes con respecto a la sexualidad y reproducción, por esta razón las adolescentes de la zona urbana y quizás aún más en la zona rural se privan de obtener acceso a información sobre los componentes esenciales de la salud sexual y reproductiva.

Este estudio pretende analizar las determinantes indirectas e intermedias relacionadas con la fecundidad adolescente y su vinculación con el nivel de escolaridad.

1 Máster en Demografía y Desarrollo, Licenciada en Trabajo Social, Directora Ejecutiva de la Asociación Acción Joven Honduras; e-mail: jinna.rosales@gmail.com 


\section{Metodología}

Este estudio se desarrolló con un enfoque cuantitativo y descriptivo, y representa una mirada al fenómeno de la fecundidad adolescente mediante la descripción cuantitativa y estadística de características demográficas de la población adolescente madre o actualmente embarazada ${ }^{2}$. Los datos estadísticos utilizados fueron los generados en la ENDESA 2011-2012 con énfasis en los principales indicadores que comprenden y explican el fenómeno de la fecundidad adolescente en Honduras.

\section{- Muestra}

La ENDESA 2011-2012 es una encuesta por muestreo realizada en 21,362 hogares y representativa a nivel nacional y departamental. La ENDESA 2011-2012 proporciona información en temas como la fecundidad, la nupcialidad, la actividad sexual, las preferencias en materia de fecundidad, el conocimiento y utilización de métodos de planificación familiar. La recolección de los datos de la ENDESA 2011-2012³ se realizó entre septiembre de 2011 y julio del 2012.

\section{- Métodos de análisis de los datos}

La información se analizó bajo el modelo de John Bongaarts $(1978,1982)^{4}$, según el modelo el máximo posible de la fecundidad representado por la tasa de fecundidad total oscila en un rango de 13 a 17 hijos por mujer. Además de la tasa de fecundidad total, la tasa global de fecundidad se encuentra básicamente influenciada por cuatro variables intermedias que son: la nupcialidad o proporción de mujeres unidas, el uso de métodos anticonceptivos, la prevalencia del aborto inducido y la infecundidad posparto (Flores, 2000, pág. 2).

Según Bongaarts, el estudio de la fecundidad respecto a sus variables intermedias se reduce a la siguiente ecuación:

\section{$\mathrm{TGF}=\mathrm{TF} * \mathrm{Cm} * \mathrm{Cc}^{*} \mathrm{Ca} * \mathrm{Ci}$}

Dónde: TGF es la tasa global de fecundidad, la cual indica el número promedio de hijos que podría tener una mujer durante su período fértil. TF es la tasa de fecundidad total o fecundidad natural, definida como la fecundidad que existiría en ausencia de un control deliberado de la natalidad. Bongaarts estimó que el nivel máximo es de aproximada- mente 15.3 hijos al final del período fértil de la mujer.

Cm es el índice de proporción mujeres unidas.

Cc es el índice de anticoncepción.

Ca es el índice de aborto inducido.

Ci es el índice de fecundidad posparto.

Cálculo de los índices Cm, Cc, Ca y Ci:

La unidad de análisis de éste estudio, son las adolescentes madres o embarazadas para el año de la encuesta. La ecuación utilizada es global de fecundidad, pero tomando en cuenta solo las adolescentes y por ende en sus respectivos cálculos y variables intermedias, de este modo se plantea la tasa global de fecundidad adolescente.

\section{Índice de proporción de mujeres unidas $(\mathrm{Cm})$ $\mathrm{Cm}=\frac{\text { Número de mujeres unidas }}{\text { Número total de mujeres }}$}

Cm será 1 si el total de las mujeres en edad fértil se encuentran unidas y 0 en el caso de que exista ausencia total de mujeres unidas. Este índice se puede calcular como el cociente entre el número total de mujeres unidas en edad fértil sobre el total de mujeres en este rango de edad (Flores, 2000, pág. 5).

\section{Índice de anticoncepción (Cc)

$$
\mathrm{Cc}=1-1.08 * u * e
$$

Dónde: $\mathrm{u}=$ Proporción de mujeres unidas que utilizan métodos anticonceptivos.

e = Efectividad promedio del método anticonceptivo. Este índice relaciona no solamente el uso de anticonceptivos, sino también la mezcla de los mismos que presenta diferentes tasas de efectividad.

Cc será 1 cuando no se utilizan métodos anticonceptivos y 0 en el caso de que todas las mujeres en edad fértil hacen uso de métodos anticonceptivos. Este indicador nos permite describir los efectos que tienen la anticoncepción y la efectividad de los métodos usados sobre la fecundidad (Flores, 2000, pág. 5).

Como las estimaciones de la efectividad de los métodos

2 Al momento de la encuesta.

3 Durante la encuesta, 22,757 mujeres de 15-49 años y 7,120 hombres de 15-59 años fueron entrevistados en los hogares seleccionados. La información recolectada es representativa a nivel nacional por lugar de residencia (urbano o rural) y para cada una de las 20 regiones de salud: 18 departamentales y dos metropolitanas. La unidad de análisis para este estudio se realizó a 5,062 mujeres adolescentes de 15 a 19 años representadas en un $22 \%$ del total de mujeres encuestadas.

4 Un modelo matemático que relaciona los determinantes próximos de la fecundidad con el nivel observado (tasa global de fecundidad), según el modelo, la diferencia en la fecundidad de las poblaciones se debe principalmente a la proporción de mujeres unidas, el uso de métodos anticonceptivos, el aborto inducido y la infecundidad por lactancia. 
son difíciles de obtener, se utilizaron los valores propuestos por la Organización Mundial para la Salud (OMS) en 2016.

\section{Índice de aborto inducido (Ca)}

En Honduras es bastante difícil obtener información acerca de cantidad de abortos debido a que en nuestro país se encuentra penalizado. Por dicho motivo, en nuestro país, este indicador es considerado igual a 1, asumiendo que no existe el aborto inducido en mujeres en edad fértil; en caso de otros países, donde sí existen registros de abortos, Ca será 1 si ninguna mujer aborta voluntariamente y 0 si todos los embarazos fueron abortados (Flores, 2000, pág. 6).

\section{Índice de infecundabilidad posparto (Ci)}

$$
C i=\frac{20}{18.5+i}
$$

Dónde: i es la duración media (en meses) de la infecundidad desde el nacimiento hasta la primera ovulación posparto; en este caso, Bongaarts considera que es algo difícil obtener este dato, así que sugiere la siguiente ecuación para la estimación de i con base en $L$, que es el tiempo medio de la duración de la lactancia exclusiva.

$$
i=1,753 \times e^{\left(0,1396 x L-0,001872 \times L^{2}\right)}
$$

Hay que tener en cuenta que, en la ecuación de índice de infecundidad posparto (Ci), el 20 corresponde al tiempo promedio en meses de intervalo entre embarazos; este surge de la suma de los meses de infecundidad por amenorrea sin lactancia (este intervalo de tiempo en promedio es de 1.5 meses), la espera para la concepción (7.5 meses), el tiempo añadido debido a muertes intrauterinas espontáneas (dos meses) y los nueve meses de gestación completa. En cuanto al 18.5, este es el período de tiempo de infecundidad posparto sin tener en cuenta el período de lactancia en que la mujer es infértil.

Ci tomará el valor de 1 en ausencia total de lactancia y 0 si la infecundidad por lactancia es para siempre, donde los factores que más influyen en la reducción del potencial de reproducción después de un nacimiento son la amenorrea posparto, la lactancia materna y la abstinencia sexual. En la medida que el período de lactancia se alarga, Ci declina (Flores, 2000, pág. 6).

\section{\|ll. Discusión de resulttados}

Para analizar las determinantes indirectas e intermedias de la fecundidad adolescente en Honduras en el período 2011-2012, se presentan algunas características sociode- mográficas de la población femenina adolescente embarazada o que al momento del levantamiento de información estaba embarazada.

Mediante la réplica del modelo de fecundidad de Bongaarts realizada en este estudio, se ha identificado cuál es el determinante próximo que más influye en la fecundidad adolescente hondureña. Del mismo modo se caracterizó cada uno de los determinantes próximos de la fecundidad que plantea el modelo de Bongaarts (nupcialidad, uso de métodos anticonceptivos, aborto inducido e infecundidad posparto) en el contexto hondureño.

\subsection{Características sociodemográficas de la población femenina adolescente que son madres o actualmente embarazadas}

Para comprender los determinantes próximos de la fecundidad es necesario profundizar en cada uno de los elementos implicados, considerando en primera instancia que la tasa de embarazo adolescente registrada en la ENDESA 2011-2012 fue del 24\%. En este sentido, caracterizar las condiciones sociodemográficas de la población femenina adolescente es crucial para la comprensión y el análisis de dicho fenómeno.

\section{- Adolescentes que son madres o actualmente embara- zadas según edades simples y área de residencia}

En su mayoría las adolescentes embarazadas o que son madres residen en el área rural, representando un 58.45\% del total de casos, lo que sugiere que el fenómeno de la fecundidad adolescente está condicionado a situaciones de pobreza, acceso a educación y recursos con los que cuenta la población, estos factores limitan las oportunidades de las adolescentes. Entre el área urbana como en el área rural se observa una diferencia de $16.92 \%$, en otras palabras, el porcentaje de madres o embarazadas en el área urbana es de $41.5 \%$.

Cuando las adolescentes inician su maternidad temprana aumenta la probabilidad de incrementar su número de hijos a lo largo de su vida reproductiva. Los datos de la Cuadro $N^{\circ} 1$ reflejan que el $13.9 \%$ de las jóvenes tenían 2 hijos y un 1\% declaró tener 3 hijos, este comportamiento tuvo mayor frecuencia entre las jóvenes del área rural con un $8.47 \%$ y un $5.49 \%$ en el área urbana.

Cuando se analizan los datos de las adolescentes que ya tienen el segundo hijo, existen coincidencias entre el área rural y urbana en relación a las edades de las adolescentes que oscilan entre los 17 y 19 años (Cuadro $\mathrm{N}^{\circ} 1$ ). 
Cuadro $\mathbb{N}^{\circ} 1$ Honduras: adolescentes que son madres o actualmente embarazadas según edades simples, número de hijos y área de residencia

\begin{tabular}{|c|c|c|c|c|c|c|c|}
\hline \multirow{3}{*}{\multicolumn{3}{|c|}{ Área de residencia }} & \multicolumn{4}{|c|}{ Número de hijos } & \multirow{4}{*}{$\begin{array}{c}\text { Total } \% \\
1.66\end{array}$} \\
\hline & & & \multirow{3}{*}{\begin{tabular}{c|}
1 \\
$\%$ \\
1.33
\end{tabular}} & \multirow{3}{*}{\begin{tabular}{c|}
2 \\
$\%$ \\
0.33 \\
\end{tabular}} & \multirow{3}{*}{$\begin{array}{l}3 \\
\% \\
0\end{array}$} & \multirow{3}{*}{$\begin{array}{l}4 \\
\% \\
0\end{array}$} & \\
\hline & & & & & & & \\
\hline \multirow{6}{*}{ Urbano } & \multirow{5}{*}{ Edades } & 15 & & & & & \\
\hline & & 16 & 6.98 & 0.42 & 0 & 0 & 7.4 \\
\hline & & 17 & 7.23 & 0.58 & 0 & 0 & 7.81 \\
\hline & & 18 & 9.55 & 1.83 & 0.07 & 0 & 11.45 \\
\hline & & 19 & 10.63 & 2.33 & 0.25 & 0 & 13.21 \\
\hline & \multicolumn{2}{|c|}{ Total } & 35.72 & 5.49 & 0.32 & 0 & 41.53 \\
\hline \multirow{6}{*}{ Rural } & \multirow{5}{*}{ Edades } & 15 & 3.16 & 0.17 & 0 & 0 & 3.33 \\
\hline & & 16 & 7.72 & 0.66 & 0 & 0 & 8.38 \\
\hline & & 17 & 11.54 & 1.41 & 0 & 0 & 12.95 \\
\hline & & 18 & 13.95 & 2.49 & 0.08 & 0 & 16.52 \\
\hline & & 19 & 12.79 & 3.74 & 0.66 & 0.08 & 17.27 \\
\hline & \multicolumn{2}{|c|}{ Total } & 49.16 & 8.47 & 0.88 & 0.08 & 58.45 \\
\hline \multirow{6}{*}{ Total } & \multirow{5}{*}{ Edades } & 15 & 4.49 & 0.50 & 0 & 0 & 4.99 \\
\hline & & 16 & 14.70 & 1.08 & 0 & 0 & 15.78 \\
\hline & & 17 & 18.77 & 1.99 & 0 & 0 & 20.76 \\
\hline & & 18 & 23.50 & 4.32 & 0.17 & 0 & 27.99 \\
\hline & & 19 & 23.42 & 6.06 & 0.91 & 0.08 & 30.47 \\
\hline & \multicolumn{2}{|c|}{ Total } & 84.88 & 13.95 & 1.08 & 0.08 & 100.00 \\
\hline
\end{tabular}

Fuente: Elaboración propia en base a datos del INE, ENDESA 2011-2012.

Cuadro $N^{\circ} 2$ Honduras: adolescentes que son madres o actualmente embarazadas según nivel de escolaridad, número de hijos y área de residencia

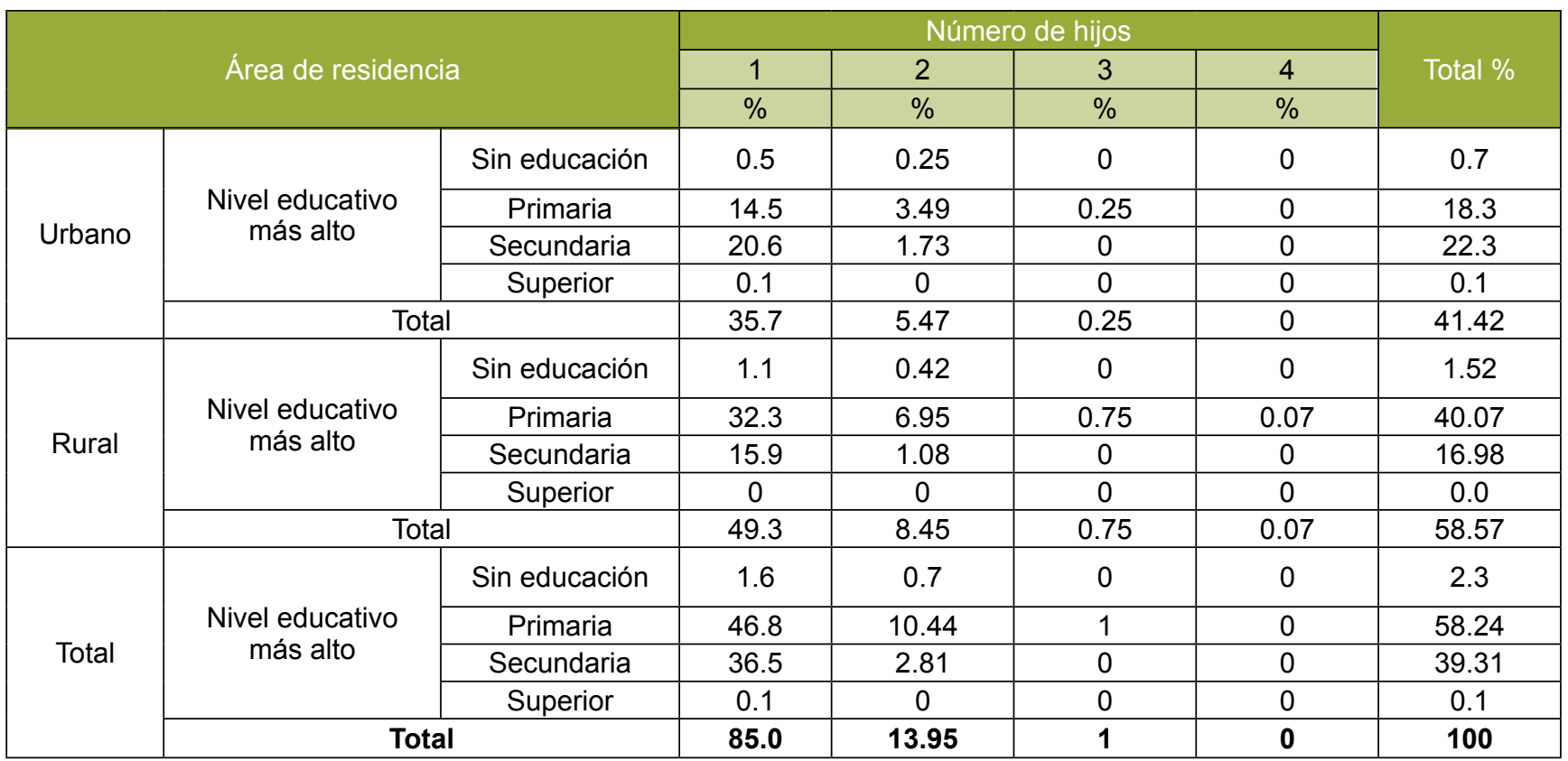

Fuente: Elaboración propia en base a datos del INE, ENDESA 2011-2012.

- Adolescentes que son madres o actualmente embarazadas según nivel de escolaridad y área de residencia

El $85 \%$ de las adolescentes madres o embarazadas para los años de la encuesta solo tenía un hijo y la mayoría de estas presentaba un nivel de educación de primaria $58 \%$ seguidas de las que manifestaron tener educación secundaria con un $39.3 \%$. Con base en este hallazgo se infiere que el nivel educativo no necesariamente influye en la prevención del embarazo en las adolescentes; de hecho, pare- 
ciera decir lo contrario cuando se observa que tan solo un $2.3 \%$ no tiene educación. Sin embargo, este hecho puede estar asociado a la edad que tienen estas adolescentes y por ende la edad escolar en la que se encuentran.

En el caso de las adolescentes que tenían 2 hijos la mayoría de ellas se ubica en el nivel de educación primaria (10.4\%) y de secundaria (2.8\%). Las adolescentes que solo tienen un hijo en el área urbana, la mayoría tiene educación secundaria y en el área rural corresponde al nivel de primaria, no obstante, cuando se observa a las adolescentes con 2 hijos en ambas áreas la mayoría tienen educación primaria, es decir que el comportamiento reproductivo de tener más hijos prevalece en aquellas adolescentes con nivel educativo de primaria. Por otro lado, esta dinámica puede ser inversa, es decir, las adolescentes no continúan sus estudios por las limitaciones que implica tener uno o más hijos, tales como: tiempo disponible, recursos económicos entre otros; sobre todo para las adolescentes del área rural (Cuadro $\mathrm{N}^{\circ} 2$ ).

\section{- Adolescentes que son madres o actualmente emba- razadas según quintil de riqueza y área de residencia}

La relación entre quintil de riqueza de las adolescentes y porcentaje de adolescentes madres o embarazadas actualmente y con más de un hijo, es casi proporcionalmente inversa; es decir, a menor ingreso, mayor número de adolescentes madres o embarazadas actualmente y con más de un hijo.

El quintil inferior, es el que más casos presenta, con excepción del segundo quintil, además de ser la población que tiene mayor número de casos con adolescentes madres que tienen más de un hijo (Cuadro $\mathrm{N}^{\circ} 3$ ). En este sentido, los recursos de los que disponen las adolescentes son un factor que influye en la fecundidad de estas, lo que se traduce como casos en los que no se previno un embarazo por la falta de acceso a métodos de planificación por las condiciones económicas de las adolescentes. Esto conlleva suponer que la fecundidad adolescente podría aumentar ya que el contexto hondureño es cada vez más desigual y con índices de pobreza altos.

Cuadro $\mathbb{N}^{\circ} 3$ Honduras: adolescentes que son madres o actualmente embarazadas según quintil de riqueza y área de residencia

\begin{tabular}{|c|c|c|c|c|c|c|c|}
\hline \multirow{3}{*}{\multicolumn{3}{|c|}{ Área de residencia }} & \multicolumn{4}{|c|}{ Número de hijos } & \multirow{4}{*}{ Total \% } \\
\hline & & & \multirow{3}{*}{\begin{tabular}{c|}
1 \\
$\%$ \\
0.83
\end{tabular}} & \multirow{3}{*}{\begin{tabular}{c|}
2 \\
$\%$ \\
0.83
\end{tabular}} & \multirow{3}{*}{$\begin{array}{c}3 \\
\% \\
0.00\end{array}$} & \multirow{3}{*}{$\begin{array}{l}4 \\
\% \\
\end{array}$} & \\
\hline & & & & & & & \\
\hline \multirow{6}{*}{ Urbano } & \multirow{5}{*}{$\begin{array}{c}\text { Índice de } \\
\text { riqueza }\end{array}$} & Quintil inferior & & & & & \\
\hline & & Segundo quintil & 5.90 & 1.16 & 0.00 & & 7.06 \\
\hline & & Quintil intermedio & 9.80 & 1.58 & 0.25 & & 11.63 \\
\hline & & Cuarto quintil & 11.46 & 1.74 & 0.08 & & 13.29 \\
\hline & & Quintil superior & 7.72 & 0.25 & 0.00 & & 7.97 \\
\hline & & Total & 35.71 & 5.56 & 0.33 & & 41.61 \\
\hline \multirow{6}{*}{ Rural } & \multirow{5}{*}{$\begin{array}{c}\text { Índice de } \\
\text { riqueza }\end{array}$} & Quintil inferior & 19.35 & 4.15 & 0.50 & 0.08 & 24.09 \\
\hline & & Segundo quintil & 16.20 & 2.49 & 0.25 & 0.00 & 18.94 \\
\hline & & Quintil intermedio & 8.64 & 1.33 & 0.08 & 0.00 & 10.05 \\
\hline & & Cuarto quintil & 3.99 & 0.50 & 0.00 & 0.00 & 4.49 \\
\hline & & Quintil superior & 1.00 & 0.08 & 0.00 & 0.00 & 1.08 \\
\hline & & Total & 49.17 & 8.55 & 0.83 & 0.08 & 58.64 \\
\hline \multirow{6}{*}{ Total } & \multirow{5}{*}{$\begin{array}{c}\text { Índice de } \\
\text { riqueza }\end{array}$} & Quintil inferior & 20.18 & 4.98 & 0.50 & 0.08 & 25.75 \\
\hline & & Segundo quintil & 22.09 & 3.65 & 0.25 & 0.00 & 26.00 \\
\hline & & Quintil intermedio & 18.44 & 2.91 & 0.33 & 0.00 & 21.68 \\
\hline & & Cuarto quintil & 15.28 & 2.24 & 0.08 & 0.00 & 17.61 \\
\hline & & Quintil superior & 8.72 & 0.25 & 0.00 & 0.00 & 8.97 \\
\hline & & Total & 84.72 & 14.04 & 1.16 & 0.08 & 100.00 \\
\hline
\end{tabular}

Fuente: Elaboración propia en base a datos del INE, ENDESA 2011-2012. 
Las características sociodemográficas de la población femenina adolescente que son madres o que estaban embarazadas, develan un contexto hondureño de desigualdad social y de género lo cual implica para las adolescentes una limitante para el desarrollo de su vida en todas las esferas de la sociedad, por otra parte, este contexto genera en las adolescentes un sometimiento ante contextos de violencia, abuso y discriminación de parte de sus parejas o de las personas que rodean su entorno social.

Ante este contexto en el que las oportunidades y una educación sexual integral se vuelve un tema de carácter urgente y de agenda pública. Muestra además la precariedad del desarrollo humano en materia de derechos de las mujeres y equidad de género, ya que desde edades tempranas las adolescentes experimentan una coartación en el goce de sus derechos, oportunidades, participación laboral, acceso a educación y su empoderamiento como mujer.

\subsection{Las variables intermedias de la fecundidad adolescente}

Con el modelo de Bongaarts ya presentado, se explica el comportamiento de la fecundidad adolescente a partir de los indicadores de sus variables intermedias, presentados en la ENDESA del año 2011-2012.

\section{- Nupcialidad}

La influencia que presenta la nupcialidad en la fecundidad adolescente se mide con la proporción de mujeres en edad fértil, que se encuentran casadas o unidas.

Desarrollando el índice de proporción de mujeres unidas $(\mathrm{Cm})$ a partir de los datos del Cuadro $\mathrm{N}^{\circ} 4$, para el período 2011-2012 hay una proporción de 0.226, es decir que el $22.6 \%$ de mujeres adolescentes en el año 2011-2012 se encontraba unida.

Cuadro $\mathbb{N}^{\circ} 4$ Honduras: total de mujeres adolescentes encuestadas y mujeres adolescentes unidas, ENDESA 2011-2012

\begin{tabular}{|c|c|c|}
\hline \multirow{2}{*}{$\begin{array}{c}\text { Grupos de } \\
\text { edad }\end{array}$} & \multicolumn{2}{|c|}{ ENDESA 2011-2012 } \\
\cline { 2 - 3 } & Mujeres unidas & Total de mujeres \\
\hline $15-19$ & $1,145(22.6 \%)$ & 5,062 \\
\hline
\end{tabular}

Fuente: Elaboración propia en base a datos del INE, ENDESA 2011-2012.

\section{- Anticoncepción}

Según la ENDESA 2011-2012 la proporción de mujeres adolescentes unidas que usan métodos anticonceptivos es de $55.7 \%$, tal como se presenta en el Cuadro $\mathrm{N}^{\circ} 5$.
Cuadro $\mathrm{N}^{\circ} 5$ Honduras: porcentaje de mujeres adolescentes que están viviendo en unión y que utilizan métodos anticonceptivos

\begin{tabular}{|c|c|c|}
\hline Método anticonceptivo actual & Casos & $\%$ \\
\hline No está usando & 507 & 44.3 \\
\hline Píldoras & 175 & 15.3 \\
\hline DIU & 39 & 3.4 \\
\hline Inyecciones & 298 & 26.0 \\
\hline Diafragma & 0 & 0.0 \\
\hline Condón & 52 & 4.5 \\
\hline Esterilización femenina & 3 & 0.3 \\
\hline Esterilización masculina & 0 & 0.0 \\
\hline Abstinencia periódica & 13 & 1.1 \\
\hline Retiro & 57 & 5.0 \\
\hline Otro & 0 & 0.0 \\
\hline Implantes & 0 & 0.0 \\
\hline Amenorrea por lactancia & 0 & 0.0 \\
\hline Condón femenino & 0 & 0.0 \\
\hline Días fijos & 1 & 0.1 \\
\hline Espuma- gel & 0 & 0.0 \\
\hline Anticonceptivo de emergencia & 0 & 0.0 \\
\hline Otro método moderno & 0 & 0.0 \\
\hline Método especifico 2 & 0 & 0.0 \\
\hline Método especifico 3 & 0 & 0.0 \\
\hline Total están Usando algún MA & 638 & 55.7 \\
\hline Total & 1145 & 100 \\
\hline
\end{tabular}

Fuente: Elaboración propia en base a datos del INE, ENDESA 2011-2012.

En relación con la efectividad de los métodos anticonceptivos, se considera el porcentaje de efectividad del 93.0\% como la media ponderante del uso de los métodos anticonceptivos más utilizados (píldoras, inyecciones y condón), como lo señala el cuadro de efectividad de los métodos anticonceptivos (según la OMS p. 70), por la población adolescente que ya está viviendo en unión.

$$
C c=1-(1.08 * 0.557 * 0.93)=0.4405
$$

De esta manera tenemos que el valor de nuestro índice de anticoncepción es de 0.4405 .

\section{- Prevalencia del aborto inducido}

Como ya ha sido sustentando en las definiciones anteriores sobre los indicadores, se asume el valor de 1 para el índice de aborto inducido, lo cual sugiere que ninguna mujer aborta voluntariamente. 


\section{- Infecundabilidad posparto}

La infecundabilidad posparto se encuentra netamente ligada a la influencia que presenta la lactancia en la disminución de la fecundidad hasta que restablezca la ovulación normal y la menstruación. El tiempo de infecundabilidad se estima con base en el tiempo medio de la lactancia.

Según el Cuadro №6, el tiempo promedio de la lactancia total para el período 2011-2012 en niños menores de tres años es de 17.0 meses para el área urbana, mientras que para la rural es de 20.5 meses. En el caso de la lactancia exclusiva, se observa que las madres en el área rural brindan mayor tiempo de lactancia exclusiva (lactancia vía pecho) que las madres del área urbana debido a que, en el área urbana, un gran porcentaje de mujeres empieza a trabajar nuevamente pocos meses después de haber tenido un hijo, a diferencia del área rural donde la gran mayoría de madres se dedica exclusivamente al cuidado y trabajo en su hogar.

Para encontrar el índice de infecundidad posparto es necesario conocer la duración mediana de la lactancia exclusiva, que para el periodo 2011-2012 es de 2.5 meses, tal como se aprecia en el Cuadro $\mathrm{N}^{\circ} 6$, esto servirá para encontrar el valor de i en la fórmula.

$\mathrm{i}=2.453$

Reemplazando 2.5 meses en L2, el valor de i será igual a 2.453 meses de duración y, por último, reemplazando este valor en la fórmula de $\mathrm{Ci}$, tendríamos:

$\mathrm{Ci}=(20) /(18.5+2.453)=0.9545$

Cuadro № 6 Honduras: Duración mediana de la lactancia materna exclusiva y de la lactancia materna predominante entre los niños viviendo con la madre nacidos en los tres años anteriores a la encuesta, Honduras 2011-2012

\begin{tabular}{|c|c|c|c|}
\hline Características & $\begin{array}{c}\text { Lactancia } \\
\text { materna } \\
\text { total }\end{array}$ & $\begin{array}{c}\text { Lactancia } \\
\text { materna } \\
\text { exclusiva } \\
\text { (sólo } \\
\text { pecho) }\end{array}$ & $\begin{array}{c}\text { Lactancia } \\
\text { materna } \\
\text { predomi- } \\
\text { nante }\end{array}$ \\
\hline \multicolumn{4}{|c|}{ Sexo } \\
\hline Hombre & 18.2 & 0.6 & 1.0 \\
\hline Mujer & 19.9 & 0.7 & 1.7 \\
\hline \multicolumn{4}{|c|}{ Residencia } \\
\hline Urbana & 17.0 & 0.6 & 0.6 \\
\hline Rural & 20.5 & 1.0 & 3.1 \\
\hline $\begin{array}{c}\text { Promedio } \\
\text { para el total } \\
\text { (2011-2012) }\end{array}$ & 18.7 & 2.5 & 3.5 \\
\hline
\end{tabular}

Fuente: Datos del INE, ENDESA 2011-2012.

\subsubsection{Tasa global de fecundidad adolescente}

\section{- Construcción del modelo}

Luego de desarrollar cada uno de los indicadores relacionados con las variables intermedias de la fecundidad, se lleva a cabo la elaboración del modelo de Bongaarts.

Se introduce como dato una estimación sobre la tasa de fecundidad natural que valora en 15.3 nacimientos por mujer (Cuadro $\mathrm{N}^{\circ} 7$ ).

Cuadro $\mathbb{N}^{\circ} 7$ Honduras: resumen de datos

\begin{tabular}{|c|c|}
\hline Resumen de datos & $2011-2012$ \\
\hline Índice de proporción de mujeres unidas Cm & 0.226 \\
\hline Índice de anticoncepción Cc & 0.4405 \\
\hline Índice de aborto inducido Ca & 1 \\
\hline Índice de infertilidad posparto Ci & 0.9545 \\
\hline Tasa de fecundidad total & 15.3 \\
\hline
\end{tabular}

Por lo que, reemplazando en la fórmula planteada por Bongaarts en su modelo, tenemos:

$T G F A=15.3 * 0.226 * 0.4405^{*} 1 * 0.9545$

$T G F A=1.45$

\section{- Comparación TGFA, método de Bongaarts y ENDESA 2011-2012}

En Honduras por cada mil mujeres adolescentes para un período de tres años ocurren en promedio, 1.45 nacimientos. Es decir, 0.48 hijos anualmente por cada mil mujeres 5 .

Al comparar la tasa global de fecundidad adolescente con el modelo de Bongaarts y el cálculo de la tasa global de fecundidad de la ENDESA, considerando únicamente la tasa específica de fecundidad en el grupo de edad de 15 y 19 años, se encontraron valores casi iguales, con un 0.50 como resultado de la división de la tasa especifica de fecundidad 101 entre 1000 por 5 en la ENDESA y un 0.48 con el modelo de Bongaarts, de este modo la comparación le confiere validez.

\section{- Influencia de las variables intermedias}

Para analizar el grado de influencia de las variables intermedias sobre la fecundidad, según el método de Bongaarts se requiere la TGFA, cuatro veces, obviando, cada vez, cada uno de los índices en el desarrollo de la fórmula, para ver qué tanto varía la TGFA.

Observando el Cuadro $\mathrm{N}^{\circ} 8$, se identifica que la variable

5 Se considera este período, ya que fue el seleccionado por la ENDESA para calcular las tasas específicas de fecundidad, incluida la tasa específica de adolescentes entre los 15 y 19 años y los datos con los cuales se desarrolló el modelo de Bongaarts en este estudio. 
con mayor grado de influencia, aplicando el método de Bongaarts, es la proporción de mujeres unidas, ya que, excluyendo esta variable del estudio de la fecundidad, la TGFA (tasa global de fecundidad adolescente) varía considerablemente, llegando a tener un valor de 6.50, es decir 6.50 hijos por adolescente, con una variación en la TGFA original de 5.04 hijos más. La segunda variable con mayor influencia en la fecundidad para el periodo 2011-2012, según el método de Bongaarts, es el uso de métodos anticonceptivos, con un valor de 3.298.

Cuadro $\mathbb{N}^{\circ} 8$ Honduras: influencia de variables intermedias

\begin{tabular}{|c|c|c|}
\hline TGFA & Nueva TGFA & $\begin{array}{c}\text { Variación respecto } \\
\text { a TGFA Bongaarts }\end{array}$ \\
\hline Sin Cm & 6.5011 & +5.0411 \\
\hline Sin Cc & 3.2987 & +1.8387 \\
\hline Sin Ca & 1.469 & +0.009 \\
\hline Sin Ci & 1.540 & +0.08 \\
\hline
\end{tabular}

Fuente: Elaboración propia en base a datos del INE, ENDESA 2011-2012

La infecundidad posparto o por lactancia también influye en la fecundidad, pero con menor grado en comparación con el uso de métodos anticonceptivos o la proporción de mujeres unidas. La variación que sufre la TGFA, sin considerarla para el desarrollo del método de Bongaarts, es de +0.08 , siendo la nueva TGFA igual a 1.5 hijos por mujer adolescente para el periodo 2011-2012.

En el caso del aborto inducido, no presenta mucha influencia en el estudio de la fecundidad, puesto que no se tienen datos exactos para su estudio, por lo que la TGF, sin su consideración, no varía mucho, sumando sólo 0.009 hijos.

\section{Conclusiones}

La fecundidad adolescente, está determinada por las cuatro variables propuestas por Bongaarts. La variable con mayor grado de influencia, es la nupcialidad, ya que, excluyendo esta variable del estudio de la fecundidad, la TGFA (tasa global de fecundidad adolescente) varía considerablemente.

La cantidad de adolescentes madres o actualmente embarazadas se concentra mayormente en el área rural, donde las adolescentes tienen menores oportunidades. En este sentido, es lógico que Honduras sea el segundo país latinoamericano con la tasa de fecundidad adolescente más alta y con altas prácticas de desigualdad de género.

La tasa global de fecundidad adolescente en el contexto hondureño es de 1.46 hijos, lo cual significa que en Honduras ocurre en promedio, por cada mil mujeres adolescentes, 1.46 nacimientos en un período de tres años.

Tomando en cuenta que el fenómeno de la fecundidad adolescente esta principalmente encausada en la nupcialidad y que a juicio de varios expertos en la temática; es un fenómeno irreversible, es importante que, instituciones y organizaciones enfoquen sus esfuerzos en la concientización de los derechos sexuales y reproductivos de las adolescentes desde un enfoque integral, para que sean capaces de tomar decisiones y disminuir el embarazo adolescente.

\section{Referencias bibliográficas}

- Adolescencia, C. R. (2013). Prevención del embarazo en la adolescencia. Pediatría Atención Primaria, vol. XV., 262.

- Arango, A. S. (2003). Niveles y determinantes regionales y subregionales de la fecundidad adolescente en Colombia . - Trabajos de grado del CIDER n. 7.

- Ávila, E. O. (2006). Factores Demográficos y Socioeconómicos de la Fecundidad Adolescente en México, 2000.

- Ávila, E. O. (2006). Factores Demográficos y Socioeconómicos de la Fecundidad Adolescente en México, 2000. San José: Ciudad Universitaria Rodrigo Facio.

- Ávila, G. A. (2011). Revista. Médica Hondureña, 68.

- Bay, G. (2003). La Fecundidad en América Latina: ¿transición o revolución? Santiago : Naciones Unidas.

- Bureau, P. R. (2013). Los Jovenes en el Mundo. El Cuadro de datos de los jóvenes en el mundo , 14.

- Busso, G. (2005). Pobreza, exclusión y vulnerabilidad social.

- Calva, L. F. (12 de Disciembre de 2013). ALC: Pobreza, baja educación y falta de oportunidades aumentan riesgo de embarazo en adolescentes. Obtenido de ALC: Pobreza, baja educación y falta de oportunidades aumentan riesgo de embarazo en adolescentes: http://www. bancomundial.org/es/news/press-release/2013/12/12/ lac-poverty-education-teenage-pregnancy 
- Castillo, M. (2007). Fecundidad adolescente en Nicaragua: tendencias, rasgos emergentes y orientaciones de política. Santiago de Chile: CEPAL - SERIE Población y desarrollo.

- CELADE/CEPAL. (2003). La Fecundidad en America Latina: ¿Transición o Revolución? . Santiago de Chile.

- CEPAL/CELADE. (1993). La Transición Demografica en America Latina.

- CEPAL/UNICEF. (2007). Maternidad Adolescete en America Latina y el Caribe, Tendencias, problemas y desafios. Boletin de la infancia y adolescencia sobre el avance de los objetivos de desarrollo del milenio, 5-9.

- Chesnais, J.-C. (1986). La transition démographique: étapes, formes, implications économiques.

- Chiarotti, N. (2009). Primera Conferencia Mundial sobre la Mujer, Mexico 1975. Instituto de Estudios Juridico Sociales de la Mujer, 1.

- Cosío, M. E. (1992). Los Antecedentes de la Transición Demografica en Mexico. En Demografía histórica II (págs. 103-128).

- Cosío, M. E. (s.f.). Los Antecedentes de la Transicion .

- Departamento de Estadística e Investigación Operativa, U. d. (s.f.). La Transición demografica.

- (2011-2012). ENDESA.

- ENDESA. (2011-2012). 81-82.

- Flores, J. B. (2000). Estudio e influencia de las variables intermedia sobre la fecundiad en el Perú: 2000. Escuela Académica Profesional de Estadística - FCM - UNMSM.

- Flores, J. B. (2000). Estudio e influencia de las variables intermedias sobre la fecundidad en el Perú: 2000. Escuela Académica Profesional de Estadística, 5.

- Fonseca, M. A. (s.f.). La transicion demográfica en Honduras. Tegucigalpa: Fondo de poblacion de las Naciones Unidas.

- Gómez, E. G. (1980). Trabajo Femenino y Fecundidad/Antecendentes de Investigación y Perpectivas Teoricas .

- Guatemala, I. N. (2013). Resumen ejecutivo del compendio estadístico sobre la situación de niñas adolescentes en Giatemala. Fondo de Población de las Naciones Unidas.

- Guiomar, B. (2003). Determinantes próximos de la fecundidad. Una aplicación a países Latinoamericanos. Santiago de Chile: CELADE.

- Hakkert, R. (2007). Un análisis del efecto de la fecundidad no deseada sobre la pobreza a nivel de los departa- mentos y zonas de residencia de Honduras 2006 . Brasilia df: UNFPA.

- Kirk, D. (1996). The demographic transition, Population Investigation Committee, Vol. 50, N.3.

- Medina, M. (2005). Trayectoria de paradigmas que explican la Fecundidad. Desarrolo y Sociedad, 81-83.

- Mendoza, M. V. (2009). factores sociodemográficos asociados con el embarazo en la adolescencia, en los departamentos de Copán, Lempira y Ocotepeque, para el año 2001. Argonautas y Caminantes, 54.

- Mendoza, M. V. (2009). Factores sociodemográficos asociados con el embarazo en la adolescencia, en los departamentos de Copán, Lempira y Ocotepeque, para el año 2001. Mendoza, M. V. (2009). factores sociodemográficos asociados con el embarazo en la adolescArgonautas y Caminantes, 53.

- Momtaz, D. (2009). La proclamación de teherán. Teherán: United Nations Audiovisual Library of International Law.

- Moreno, E. E. (2006). “Determinantes próximos de la fecundidad en Haití (1995-2000)". Mexico.

- OPS, M. M. (1995). La salud adolescente y del joven. Washington D. C., E.U.A: OPS.

- (2015). Política Nacional de Salud Sexual y Reproductiva.

- Republica.net, L. (19 de Febrero de 2018). Embarazo adolescente en Centroamérica es mayor al promedio mundial. Embarazo adolescente en Centroamérica es mayor al promedio mundial, pág. 1.

- Roustan, J. A. (1995). Bongaarts: un modelo de fecundidad y su aplicacion en España. Boletin de la asociacion de demografia historica, XIII, 3, 1995, 86.

- Salvador, M. d. (2014). "Situación de la Niñez y Adolescencia en El Salvador 2014. Fondo de Población de las Naciones Unidas.

- Sitema de Estadisticas Sociodemograficas /Area de educación . (s.f.).

- Social, F. N. (Octubre 2016). Causas y consecuencias económicas y sociales del embarazo adolescente en Nicaragua. FUNIDES.

- Valdés. (2000). “Población reto del tercer milenio: Curso interactivo introductoria a la demografía". Mexico D.F.

- Vignoli, J. R. (2003). La Fecundidad alta en el Istmo Centroamericano: Un Riesgo en Transición. Costa Rica. 
- Vignoli, J. R. (2012). Reproduccion Temprana en Centroamerica: escenarios emergentes y desafios. Naciones Unidas .

- Zelaya, M. (2014). Condiciones de vida familiar y conductas reproductivas:. Demomujer, 27-28. 